\title{
ESTRUTURA DE SOLOS EM MANEJO CONSERVACIONISTA: DIAGNÓSTICO VISUAL, LABORATORIAL, CARACTERIZAÇÃO E INTER-RELAÇÕES
}

Soil structure in conservationist management: diagnosis visual, laboratory, characterization and inter-relations

\author{
Érika Andressa da Silva ${ }^{1 *}$; Carla Eloize Carducci²; Geraldo César de Oliveira ${ }^{3}$; Bruno Montoani Silva ${ }^{4}$; Milson Evaldo \\ Serafim ${ }^{5}$ \\ ${ }^{1}$ Doutoranda em Ciência do Solo; Departamento de Ciência do Solo; Universidade Federal de Lavras; \\ andressaerikasilva@gmail.com \\ ${ }_{2}^{2}$ Profa. Dra; Faculdade de Ciências Agrárias; Universidade Federal da Grande Dourados; elocarducci@hotmail.com \\ 3Prof. Dr; Departamento de Ciência do Solo; Universidade Federal de Lavras; geraldooliveira@dcs.ufla.br \\ 4Prof. Dr; Departamento de Ciência do Solo; Universidade Federal de Lavras; montoani@gmail.com \\ 5Prof. Dr; Instituto Federal de Educação, Ciência e Tecnologia de Mato Grosso - IFMT, Campus Cáceres; \\ milson.serafim@cas.ifmt.edu.br
}

Artigo enviado em 07/04/2017, aceito em 05/07/2017 e publicado em 29/09/2017.

Resumo - A análise visual da estrutura do solo pode ser uma ferramenta útil e prática no monitoramento da condição física do solo em sistemas de manejo conservacionista. Assim, o objetivo deste trabalho foi analisar a relação entre o diagnóstico visual e laboratorial da estrutura do solo, em sistema de manejo conservacionista. Utilizou-se delineamento experimental inteiramente casualizado em esquema fatorial duplo, sendo os fatores as classes de solos (CAMBISSOLO HÁPLICO, LATOSSOLO VERMELHO e NITOSSOLO HÁPLICO), avaliadas em diferentes profundidades (amontoa $[-0,05-0,15 \mathrm{~m}] ; 0,05-0,15 \mathrm{~m} ; 0,25-0,35 \mathrm{~m}$; e $0,45-0,55 \mathrm{~m}$ ), em triplicata. A avaliação se apoiou na aparência visual da estrutura dos solos após 5 anos da implantação de um sistema de manejo conservacionista, sendo avaliadas a resistência, consistência, porosidade e presença de raízes nas unidades estruturais dos solos, categorizados, segundo a carta padrão, por 5 escores visuais (Ev) utilizados na classificação da qualidade $\mathrm{da}$ estrutura, variando de $\mathrm{Ev}=1$ (melhor qualidade) a $\mathrm{Ev}=5$ (pior qualidade). Os atributos físicos do solo (macroporosidade, microporosidade, distribuição de poros por tamanho e densidade do solo), foram determinados em amostras indeformadas submetidas aos potenciais matriciais de -2, -4, -6, -10 kPa nas unidades de sucção, e -33, 100, -500, $-1500 \mathrm{kPa}$ nas câmaras de Richards. A agregação foi avaliada pelo diâmetro médio geométrico. Nas profundidades de 0,25-0,35 m e 0,45-0,55 m, o Latossolo apresentou Ev menor quando comparado ao Nitossolo e Cambissolo, indicando sua melhor condição estrutural nestas profundidades. O aumento dos Ev está relacionado à perda da porosidade estrutural (interagregados) e incremento da porosidade textural (intraagregados). A análise de componentes principais demonstrou que a quantificação dos atributos densidade do solo, microporosidade, teor de silte e poros da classe de diâmetro $<0,6 \mu \mathrm{m}$ contribuíram para justificar o aumento do Ev.

Palavras-chave - análise visual, atributos físicos, manejo do solo.

Abstract - The visual analysis of soil structure can be a useful and practical tool for monitoring soil physical condition in conservation management systems. Thus, the objective of this work was to analyze the relationship between visual and laboratorial diagnosis of soil structure, in a conservation management system. We utilized completely randomized experimental design in a factorial double was used, the factors being soil classes (Haplic Cambisol, Red Latosol and Haplic Nitosol), evaluated at different depths (pille [-0.05-0.15 m]; 0.05-0.15 m; 0.25-0.35 $\mathrm{m}$ and $0.45-0.55 \mathrm{~m}$ ) in triplicate. The evaluation was based on the visual appearance of the structure of the soil after five years of implementation of a management system considered conservationist, being analyzed resistance, consistency, porosity and presence of roots in the structural units of the soils, categorized by five visual scores (Ev) used in quality grading structure, ranging from $\mathrm{Ev}=1$ (best quality) at $\mathrm{Ev}=5$ (worst quality). In order to evaluate the physical attributes of the soil (macroporosity, microporosity, pore distribution by size and soil density), undisturbed samples were collected and submitted to matrix potentials $-2,-4,-6,-10 \mathrm{kPa}$ in the suction units, and $33,-100,-500,-1500 \mathrm{kPa}$ in the Richards chamber. The aggregation was evaluated by the geometric mean diameter. We observed in the depths of $0.25-0.35$ and $0.45-0.55 \mathrm{~m}$, the Latosol presented visual score lower when compared to Nitosol and Cambisol, indicating its better structural condition in these depths, comparative form to other soils analyzed. The increase of the visual scores is related to the loss of the structural porosity (inter aggregates) and increase of the textural porosity (intraaggregates). The principal component analysis demonstrated that quantification of the attributes density, microporosity, silt content and the pore diameter class $<0.6 \mu \mathrm{m}$ helped to justify the increase in Ev.

Keywords - physical attributes, soil management, visual analysis. 


\section{INTRODUÇÃO}

Os métodos laboratoriais são importantes ferramentas para subsidiar a escolha de um determinado sistema de manejo do solo, especialmente em pesquisas científicas. No entanto, é necessário o desenvolvimento de metodologias de diagnóstico rápido e confiável da qualidade estrutural do solo, que permitam ao extensionista ou produtor rural, a obtenção de informações auxiliares para tomadas de decisão. Atendendo a estas premissas foram desenvolvidas técnicas de análise visual que permitem diagnosticar a estrutura do solo (GUIMARÃES et al., 2011).

Todavia, a subjetividade de alguns métodos de análise visual tem sido apontada como o principal motivo de crítica, pois se baseia na observação da estrutura do solo direcionada por meio de tabelaspadrão que identificam, por exemplo, os níveis de agregação, presença de camadas compactadas, ou mesmo atividade biológica mais intensa (GUIMARÃES et al., 2011).

Com o intuito de diminuir a subjetividade das técnicas de diagnóstico visual empregadas no campo, metodologias têm sido propostas, testadas e modificadas (BALL et al., 2007; GUIMARÃES et al., 2011; JOHANNES et al., 2016). Atualmente, o método de Avaliação Visual da Qualidade da Estrutura do Solo (AVES), adaptado por Ball et al. (2007), é reconhecido como um dos mais eficazes indicadores da qualidade estrutural do solo em regiões temperadas, por permitir o exame detalhado e o julgamento quantificado dos aspectos da estrutura.

Em solos tropicais, algumas limitações à aplicação desta técnica foram referenciadas em alguns trabalhos. A AVES é realizada em blocos de solo, cuja extração, em função do tamanho da amostra ou da umidade do solo, pode ser dificultada, e a necessidade de abertura de trincheiras para coleta destes blocos pode ser muito onerosa (GIAROLA et al., 2009), principalmente, para condução de avaliações em solos sob sistemas de manejo com culturas perenes, em que são necessárias coletas em camadas mais profundas.

Alguns critérios que contribuem para a definição do escore são o tamanho, a aparência e o grau de resistência dos agregados (BALL et al., 2007; GUIMARÃES et al., 2011). Assim, os níveis de agregação que são avaliados pela quebra dos agregados nas suas linhas de fraqueza também podem ser influenciados pela umidade atual no momento da análise (JOHANNES et al., 2016).

A aplicação da técnica exige conhecimentos prévios a respeito de morfologia da estrutura do solo (GIAROLA et al., 2009). Portanto, um treinamento se faz necessário para uma correta interpretação das informações resultantes da AVES.
O método de AVES teve sua importância consolidada ao permitir uma definição em campo das profundidades mais relevantes para amostragens (GIAROLA et al., 2009; PRICE et al., 2013). Pois, desta forma, a escolha dos locais para coleta de amostras e as determinações laboratoriais passam a ser referenciadas, e a possibilidade de acerto no julgamento da qualidade estrutural do solo tende a ser maior (PRICE et al., 2013).

Em recente estudo, Johannes et al.(2016) demonstraram que a AVES pode ser comparada ou associada a propriedades físicas, como estabilidade de agregados, índice $\mathrm{S}$, intervalo hídrico ótimo, resistência à penetração (PRICE et al., 2013; GUIMARÃES et al., 2013; MONCADA et al., 2014) e variabilidade espacial da estrutura do solo (organização dos sólidos e vazios) Carducci et al. (2016).

Todavia, a densidade do solo é a propriedade mais representada nessas comparações (JOHANNES et al., 2016). Em associação com análises visuais, a densidade do solo, tem contribuído para interpretação mais global dos problemas verificados no campo e tem facilitado o entendimento das influências do manejo do solo em sua estrutura, em especial nos processos de infiltração da água, de erosão e, principalmente, no desenvolvimento das raízes das plantas (MONCADA et al., 2014).

Guimarães et al. (2013) citam que correlações entre escore visual (julgamento ou nota) e atributos físicos do solo medidos em laboratório, apoiam o uso de análises de campo como um método semiquantitativo confiável para determinar algumas propriedades chaves do solo, particularmente aquelas envolvidas na resposta a mudanças da estrutura do solo devido ao uso e manejo.

Em regiões como o cerrado, que apresenta uma estação seca marcante, e cujas variações topográficas, permitem a ocorrência de distintas classes de solos, dos mais rasos (Cambissolos) aos mais profundos (Latossolos), a AVES, pode ser uma ferramenta valiosa para subsidiar de forma rápida a escolha de práticas de manejo mais adequadas a cada tipo de solo.

Em condições naturais, sob mata nativa, a AVES tem indicado pior qualidade estrutural de solos pouco profundos, como Cambissolos, ao confirmar a presença de agregados mais coesos, profundidades adensadas ou compactadas e baixa porosidade visível. Por outro lado, nos Latossolos, pela AVES tem se observado elevada porosidade visível, intensa atividade biológica e bom estado de agregação, não ocorrendo limitações físicas ao desenvolvimento das plantas (GIAROLA et al., 2009; 2010).

A AVES tem se apresentado como ferramenta eficiente para a avaliação dos efeitos de sistemas de manejo conservacionistas, como plantio 
direto, na estrutura dos solos (GIAROLA et al., 2010, 2013). Desta forma, este método pode ser eficiente para o diagnóstico da qualidade estrutural de solos sob outros sistemas de manejo conservacionistas, como o empregado na cafeicultura na região do Alto São Francisco, em Minas Gerais.

Nesse sistema de manejo na cafeicultura, o condicionamento físico é obtido com preparo profundo do solo (sulcamento a $60 \mathrm{~cm}$ de profundidade) seguido de correções químicas e fertilização, além da aplicação, em superfície, de doses de gesso agrícola não convencionais, superiores àquelas recomendadas na literatura (SERAFIM et al., 2011; SERAFIM et al., 2013).

A manutenção de braquiária nas entre linhas da cultura é outro ponto de grande relevância nesse sistema. Essa gramínea, nos primeiros 2 anos, é manejada com cortes periódicos e todo resíduo vegetal proveniente dos cortes é direcionado para a linha de plantio, constituindo-se em aporte contínuo de matéria orgânica para o solo. O sistema de manejo também preconiza a adoção de terraços em patamar nas áreas mais declivosas que, associado à cobertura vegetal nas entre linhas, faz dele um eficiente sistema conservacionista do solo (SILVA et al., 2016a,b).

Este sistema de manejo vem sendo empregado em latossolos, cambissolos e nitossolos mas, pesquisas científicas que respaldem sua recomendação para diferentes solos são muito incipientes. Em adição, análises visuais da qualidade estrutural de solos sob o referido sistema de manejo ainda não foram realizadas. Assim, este trabalho teve por objetivo analisar a relação entre análise visual e laboratorial da estrutura objetivando diagnóstico da qualidade física de diferentes solos cultivados com cafeeiro em sistema de manejo conservacionista.

\section{MATERIAL E MÉTODOS}

\section{Descrição da área experimental}

A pesquisa foi realizada em solos sob lavouras cafeeiras comerciais com cinco anos de cultivo, implantados segundo as premissas de um sistema de manejo do solo que emprega práticas conservacionistas tais como: 1- mecânicas: construção de miniterraços na base da planta; 2- vegetativa: cultura de cobertura na entrelinha do cafeeiro (braquiária); 3- edáfica: correção química do solo em área total e fertilização dos sulcos de plantio (SERAFIM et al., 2011; CARDUCCI et al., 2015).

Este sistema de manejo vem sendo utilizado nos municípios de São Roque de Minas e Vargem Bonita, na Região do Alto São Francisco (MG). O clima da região é do tipo Cwa, segundo classificação de Köppen. A precipitação média anual é de $1.344 \mathrm{~mm}$, com estação seca bem definida nos meses de maio a setembro. A temperatura média anual é de $20,7^{\circ} \mathrm{C}$, umidade relativa média de $60 \%$ e altitude média de 900 m (MENEGASSE et al., 2002).

Os solos em estudo foram classificados segundo Santos et al. (2013) como CAMBISSOLO HÁPLICO, LATOSSOLO VERMELHO e NITOSSOLO HÁPLICO, sendo o primeiro de textura argilosa e os dois últimos de textura muito argilosa (Tabela 1).

Tabela 1 - Granulometria* do Cambissolo Háplico, Latossolo Vermelho e Nitossolo Háplico em sistema de manejo conservacionista.

\begin{tabular}{cccc}
\hline Horizontes & Areia & Silte & Argila \\
\hline \multicolumn{4}{c}{$\ldots \ldots \ldots . . . \mathrm{g} \mathrm{kg}^{-1} \ldots \ldots \ldots \ldots}$. \\
Ap & 123 & 337 & 540 \\
$\mathrm{Bi}$ & 50 & 432 & 518 \\
& LATOSSOLO VERMELHO \\
Ap & 32 & 118 & 850 \\
$\mathrm{Bw}$ & 37 & 96 & 867 \\
& NITOSSOLO HÁPLICO & \\
Ap & 90 & 141 & 769 \\
$\mathrm{Bt}$ & 79 & 140 & 781 \\
\hline
\end{tabular}

*Método da pipeta (Embrapa, 2011).

Estes solos são originados de rochas pelíticas, principalmente siltitos. Anteriormente, a implantação das lavouras comerciais, as áreas de Latossolo, Cambissolo e Nitossolo eram cobertas por pastagem com Brachiaria decumbens, que foi implantada após supressão da vegetação nativa de Cerrado (savana brasileira) em 2007.

Os solos foram preparados entre os meses de Julho e Agosto de 2008, com uma aração e duas gradagens, conforme manejo tradicional da região, além da correção química, incorporando, independente do solo, $4 \mathrm{Mg} \mathrm{Ma}^{-1}$ de calcário (16-17\% $\mathrm{MgO}$ ) em área total, e posteriormente aplicados $2 \mathrm{Mg} \mathrm{ha}^{-1}$ de gesso agrícola em superfície.

$\mathrm{Na}$ segunda quinzena de outubro de 2008, foi feito o plantio do cafeeiro (Coffea arábica L.), cultivar Catucaí Amarelo, no espaçamento semi-adensado 2,50 x 0,65 m. Para o plantio, foi feito sulcamento com cavadeira-adubadora que prepara a $0,60 \mathrm{~m}$ de profundidade e $0,50 \mathrm{~m}$ de largura, e promove mistura de corretivos e fertilizantes em toda a extensão do sulco. No sulco de plantio foram aplicadas adicionalmente $2 \mathrm{Mg} \mathrm{ha}^{-1}$ de calcário (16-17\% MgO) e $220 \mathrm{~kg} \mathrm{~m}^{-1}$ da formulação NPK 08-44-00 com 1,5\% Zn e $0,5 \% \mathrm{~B}$, visando construir a fertilidade na camada de 0,60 m (SERAFIM et al., 2011, 2013). A caracterização química para os solos antes da implantação do sistema de manejo e após 5 anos estão na tabela 2. 
REVISTA SCIENTIA AGRARIA

Versão On-line ISSN 1983-2443

Versão Impressa ISSN 1519-1125

SA vol. $18 \mathrm{n}^{\circ} .3$ Curitiba Jul/Set. 2017 p. 61-73

Tabela 2 - Caracterização química de Cambissolo, Latossolo e Nitossolo antes e após cinco anos da implantação do sistema de manejo.

\begin{tabular}{|c|c|c|c|c|c|c|c|}
\hline \multirow{3}{*}{ Solo } & Antes da in & tação do sis & de manejo & Após 5 an & da implant: & o do sistem & de manejo \\
\hline & \multicolumn{7}{|c|}{ Camadas (m) } \\
\hline & $0,05-0,15$ & $0,25-0,35$ & $0,45-0,55$ & Amontoa & $0,05-0,15$ & $0,25-0,35$ & $0,45-0,55$ \\
\hline \multirow[b]{2}{*}{ Cambissolo } & \multicolumn{7}{|c|}{ 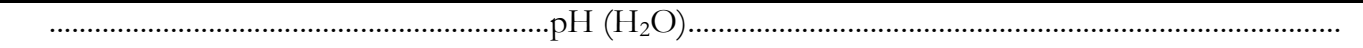 } \\
\hline & 4,90 & 5,20 & 5,40 & 3,80 & 4,23 & 4,57 & 4,73 \\
\hline Latossolo & 4,40 & 4,70 & 4,58 & 4,00 & 4,23 & 4,33 & 4,50 \\
\hline \multirow[t]{2}{*}{ Nitossolo } & 4,60 & 4,70 & 4,90 & 5,30 & 4,87 & 4,63 & 4,60 \\
\hline & \multicolumn{7}{|c|}{... $\mathrm{K}^{+}\left(\mathrm{mg} \mathrm{dm}^{-3}\right) \ldots$} \\
\hline Cambissolo & 162,60 & 41,30 & 28,67 & 100,67 & 206,00 & 104,67 & 59,33 \\
\hline Latossolo & 73,33 & 38,67 & 56,00 & 84,67 & 37,33 & 35,33 & 29,33 \\
\hline \multirow[t]{2}{*}{ Nitossolo } & 74,00 & 47,30 & 36,00 & 234,67 & 52,67 & 23,33 & 23,33 \\
\hline & \multicolumn{7}{|c|}{. } \\
\hline Cambissolo & 0,50 & 0,10 & 0,10 & 6,23 & 4,33 & 2,30 & 1,80 \\
\hline Latossolo & 0,10 & 0,10 & 0,10 & 3,50 & 5,73 & 4,80 & 2,17 \\
\hline \multirow[t]{2}{*}{ Nitossolo } & 0,10 & 0,13 & 0,10 & 4,70 & 3,60 & 3,57 & 3,00 \\
\hline & \multicolumn{7}{|c|}{ 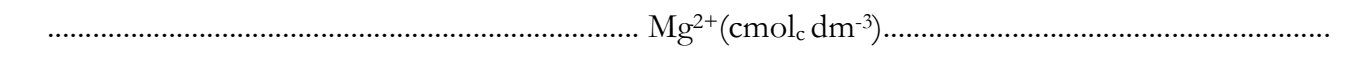 } \\
\hline Cambissolo & 0,47 & 0,10 & 0,10 & 0,10 & 0,20 & 0,10 & 0,10 \\
\hline Latossolo & 0,10 & 0,10 & 0,10 & 0,10 & 0,10 & 0,20 & 0,20 \\
\hline \multirow[t]{2}{*}{ Nitossolo } & 0,10 & 0,10 & 0,10 & 0,93 & 0,13 & 0,10 & 0,10 \\
\hline & \multicolumn{7}{|c|}{.......... $\mathrm{Al}^{3+}\left(\mathrm{cmol}_{\mathrm{c}} \mathrm{dm}^{-3}\right) \ldots$} \\
\hline Cambissolo & 1,40 & 1,37 & 0,97 & 2,30 & 0,77 & 0,63 & 0,50 \\
\hline Latossolo & 3,60 & 2,67 & 2,23 & 0,17 & 0,17 & 0,23 & 0,20 \\
\hline \multirow[t]{2}{*}{ Nitossolo } & 1,37 & 0,87 & 1,12 & 2,63 & 1,50 & 1,13 & 0,90 \\
\hline & \multicolumn{7}{|c|}{. CTC potencial $\left(\mathrm{cmol}_{\mathrm{c}} \mathrm{dm}^{-3}\right)$} \\
\hline Cambissolo & 9,25 & 5,18 & 3,51 & 19,83 & 13,86 & 7,37 & 5,42 \\
\hline Latossolo & 10,21 & 8,17 & 9,19 & 19,21 & 17,39 & 15,31 & 8,99 \\
\hline \multirow[t]{2}{*}{ Nitossolo } & 15,18 & 12,20 & 9,78 & 11,88 & 9,31 & 10,03 & 8,80 \\
\hline & \multicolumn{7}{|c|}{ 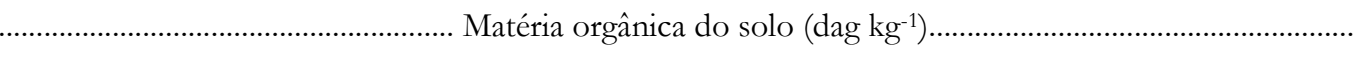 } \\
\hline Cambissolo & 3,89 & 1,00 & 0,65 & 3,18 & 3,56 & 1,29 & 0,65 \\
\hline Latossolo & 3,89 & 2,96 & 3,43 & 3,70 & 3,32 & 3,24 & 1,83 \\
\hline Nitossolo & 3,75 & 2,71 & 1,79 & 4,19 & 3,56 & 2,70 & 2,53 \\
\hline
\end{tabular}

No início de 2009 foram adicionados outros 7 $\mathrm{kg}$ de gesso agrícola/metro linear na linha do plantio de cafeeiros. Quando a braquiária da entre linha atingiu 0,50 $\mathrm{m}$ de altura, o solo foi gradeado a $0,10 \mathrm{~m}$ de profundidade e este material vegetal foi amontoado junto ao tronco do cafeeiro numa faixa de $0,5 \mathrm{~m}$, configurando uma amontoa sobre o gesso adicionado na superfície do solo na linha do cafeeiro (SERAFIM et al., 2011).

No primeiro ano após o plantio, foram aplicados, em área total, $100 \mathrm{~kg} \mathrm{ha}^{-1}$ de nitrato de amônio em cobertura. No segundo ano de cultivo, objetivando o bom rendimento de material cortado e incremento de material orgânico, o solo recoberto com a gramínea (entre linhas) recebeu uma adubação com $600 \mathrm{~kg} \mathrm{ha}^{-1}$ de $\mathrm{K}_{2} \mathrm{O}$, fracionada em 4 aplicações durante o ano (SILVA et al., 2016a,b).
A partir do terceiro ano, as correções químicas do solo foram realizadas de acordo com a interpretação das análises de solo pela quinta aproximação (GUIMARÃES et al., 1999). Todos os anos de cultivo foram realizadas análises nutricionais e quando necessário, micronutrientes foram aplicados via adubação foliar.

Após dois anos, os cafeeiros estão estabelecidos e sombreiam a braquiária das entrelinhas. Assim, o aporte de material vegetal na linha é significativo nos dois primeiros anos, em que se tem a necessidade de controle da braquiária, a fim de evitar sua competição com a cultura principal. Os resíduos vegetais de braquiária colocados na linha de cultivo, ao longo dos dois primeiros anos, são essenciais para a estruturação do solo, que foi revolvido à $0,60 \mathrm{~m}$. 


\section{Análises laboratoriais}

Após a realização das análises visuais, foram coletadas amostras com estrutura preservada para determinação de atributos físicos do solo. Os anéis volumétricos de altura $0,025 \mathrm{~m}$ foram posicionados no centro das profundidades de amontoa; 0,05-0,15 m; $0,25-0,35 \mathrm{~m}$ e $0,45-0,55 \mathrm{~m}$. A amontoa refere-se a uma profundidade de $-0,05-0,15 \mathrm{~m}$ (acima da superfície do solo), conforme figura 1.

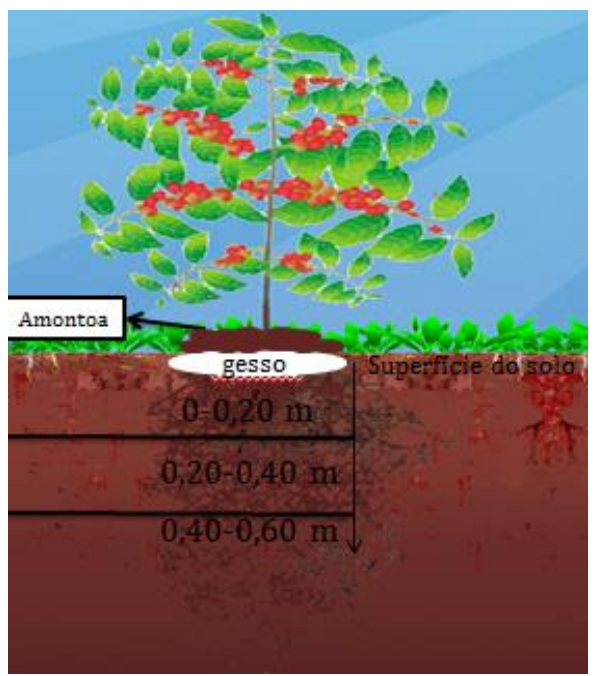

Figura 1 - Esquema ilustrativo das camadas do solo sob manejo.

Para determinação das curvas de retenção de água (CRA), as amostras com estrutura preservada foram inicialmente saturadas gradualmente e submetidas aos potenciais matriciais $(\Psi \mathrm{m})-2,-4,-6$, $10 \mathrm{kPa}$ utilizando as unidades de sucção, e $-33,-100$, $500,-1500 \mathrm{kPa}$ nos extratores de placas porosas. Em seguida, as amostras foram secas em estufa, a 105-110 ${ }^{\circ} \mathrm{C}$, por $48 \mathrm{~h}$, para a determinação do conteúdo de água correspondente aos $\Psi \mathrm{m}$ e densidade do solo (Ds) (EMBRAPA, 2011).

Posteriormente, objetivando a obtenção das curvas de retenção de água do solo foi ajustado o modelo de van Genuchten (1980) com restrição de $\mathrm{m}=1-1 / \mathrm{n}$ (MUALEM, 1976), aos dados experimentais. Para a quantificação do diâmetro de poros, foi utilizada a equação proposta por Bouma (1973):

\section{$\mathrm{D}=4 \sigma \operatorname{Cos} \theta / \Psi \mathrm{m}$}

Onde: D é o diâmetro do poro $(\mu \mathrm{m}) ; \sigma$ a tensão superficial da água $\left(73,43 \mathrm{kPa}\right.$ a $\left.20^{\circ} \mathrm{C}\right) ; \theta$ é o ângulo de contato entre o menisco e a parede do tubo capilar (considerado como zero) e $\Psi \mathrm{m}$ o potencial matricial $(\mathrm{kPa})$. O diâmetro do poro foi relacionado a cada $\Psi \mathrm{m}$ determinado da CRA e aplicado às amostras.
A microporosidade foi considerada como sendo a água retida no solo em $\Psi \mathrm{m}-6 \mathrm{kPa}$, e a macroporosidade calculada pela diferença entre porosidade total $(\mathrm{PT}=(1-\mathrm{Ds} / \mathrm{Dp})) \mathrm{e}$ microporosidade (EMBRAPA, 2011). A densidade de partículas foi determinada pelo método do picnômetro (EMBRAPA, 2011). A estabilidade de agregados foi determinada por peneiramento úmido (MAZURAK, 1950) e expressa pelo índice: diâmetro médio geométrico. A matéria orgânica do solo foi determinada de acordo com Embrapa (2009). Os dados de produtividade das lavouras em cada classe de solo estudada foram obtidos considerando-se a produção total de cada área.

\section{Análise visual da estrutura do solo}

As avaliações visuais da qualidade da estrutura foram realizadas a campo com o auxílio da carta padrão de determinação da qualidade da estrutura do solo (BALL et al., 2007). Foram abertas trincheiras até a profundidade de $1,5 \mathrm{~m}$, nas três classes de solo, e adotou-se como referência para coleta dos blocos, o Perfil Cultural (TAVARES FILHO et al., 1999) da ordem de solo que apresentou condições mais restritivas ao desenvolvimento do sistema radicular.

Desta forma, as profundidades coletadas, em quatro repetições, foram definidas com base no Perfil Cultural do Cambissolo, em que foram identificadas 4 profundidades com unidades estruturais homogêneas (amontoa, 0,0-0,20 m; 0,20-0,40 m, 0,40-0,60 m), que diferiram entre si, pelo formato e tamanho dos agregados.

No momento da coleta de solo, verificou-se, na superfície do solo que se encontrava recoberta pela amontoa, a presença de uma camada de gesso residual $(\sim 1 \mathrm{~cm})$. Buscando-se evitar a amostragem desse gesso, a coleta dos blocos $(0,10 \times 0,10 \times 0,05 \mathrm{~m})$ iniciou-se a uma distância vertical de $5 \mathrm{~cm}$ a partir da linha de gesso. Para a amostragem nas demais camadas $(0,20$ 0,40; 0,40-0,60 m), empregou-se o mesmo critério, 5 $\mathrm{cm}$ após a marcação do limite entre os volumes homogêneos. Em função disso, os blocos foram posicionados com o seu centro alinhado ao da profundidade, o que definiu as camadas de amostragem da seguinte forma: amontoa $(-0,05-0,15 \mathrm{~m}) ; 0,05-0,15$ m; 0,25-0,35 m; e 0,45-0,55 m.

Salienta-se que pelos critérios descritos na metodologia de analise visual, quando dentro do bloco há distintas camadas, deve-se se utilizar a média ponderada das mesmas para descrever o escore do bloco (GIAROLA et al., 2010; GUIMARÃES et al., 2011). Entretanto, neste trabalho esta situação não foi observada e adotou-se a média de todo o volume, conforme o método tradicional (BALL et al., 2007). Após coletados, os blocos foram colocados sobre uma superfície plana, e o solo do bloco foi retirado com 
extremo cuidado, buscando evitar a desagregação do solo fora das linhas de ruptura natural. Em Seguida foram tiradas fotografias para a comparação com a carta de avaliação visual do método desenvolvida por Ball et al. (2007) e adaptada por Guimarães et al. (2011).

A avaliação da estrutura se apoiou na aparência, resistência, consistência, porosidade visível e presença de raízes nas unidades estruturais dos solos conforme proposto por Ball et al.(2007), e cada profundidade de solo foi classificada em uma das cinco categorias de escores visuais (Ev) propostas em Guimarães et al. (2011) em que $\mathrm{Ev}=1$ ou 2 foram considerados como solos de estrutura de boa qualidade, que não requerem mudanças no manejo, Ev $=3$ solo de qualidade estrutural adequada, mas com necessidade de alterações no manejo de forma a evitar perda de qualidade estrutural, e $E v=4$ ou 5 foram considerados solos de baixa qualidade estrutural, exigindo mudanças no manejo do solo.

\section{Análises estatísticas}

Utilizou-se do delineamento experimental inteiramente casualizado em esquema fatorial duplo, sendo os fatores as classes de solo (Latossolo, Cambissolo e Nitossolo) e as profundidades avaliadas (amontoa: -0,05-0,15 m; 0,05-0,15 m;0,25-0,35 m; e
0,45-0,55 m), em triplicata. Para os dados de atributos físicos as inferências estatísticas foram feitas ao nível de $5 \%$ de probabilidade de erro, tendo sido as médias comparadas pelo teste de Scott-Knott, por meio do aplicativo computacional SISVAR (FERREIRA, 2011). Aos dados qualitativos (notas de escore visual) foi aplicado o teste não paramétrico de Kruskal-Wallis (P $<0,05)$ que permitiu comparações múltiplas dos solos (dois a dois). Análises de correlação foram realizadas com auxílio do sistema computacional Sigma Plot e visando discriminar as variáveis melhor correlacionadas com o Ev, procedeu-se à análise de componentes principais (ACP), por meio do software $\mathrm{R}$ ( $\mathrm{R}$ Development Core Team, 2013).

\section{RESULTADOS E DISCUSSÃO}

\section{Propriedades físicas do solo}

Os valores de densidade do solo, microporosidade, macroporosidade e porosidade total para os solos estudados na profundidade de amontoa e nas profundidades de $0,05-0,15 \mathrm{~m}, 0,25-0,35 \mathrm{~m}, 0,45-$ $0,55 \mathrm{~m}$, estão apresentados na Tabela 3 .

Tabela 3 - Valores de densidade do solo, microporosidade, macroporosidade e volume total de poros no perfil de solos sob cultivo de cafeeiro em sistema de manejo conservacionista.

\begin{tabular}{|c|c|c|c|}
\hline \multirow[t]{2}{*}{ Profundidade $(\mathrm{m})$} & Cambissolo & Latossolo & Nitossolo \\
\hline & \multicolumn{3}{|c|}{ Densidade do solo ${ }^{* * *}\left(\mathrm{Mg} \mathrm{m}^{-3}\right)$} \\
\hline Amontoa $(-0,05-0,15)$ & $0,95 \mathrm{~A} \mathrm{c}$ & $0,83 \mathrm{~A} \mathrm{a}$ & $0,94 \mathrm{~A} \mathrm{c}$ \\
\hline $0,05-0,15$ & $0,96 \mathrm{~B} \mathrm{c}$ & $0,92 \mathrm{~B}$ a & $1,13 \mathrm{~A} \mathrm{a}$ \\
\hline $0,25-0,35$ & $1,04 \mathrm{~A} \mathrm{~b}$ & $0,98 \mathrm{~A} \mathrm{a}$ & $1,03 \mathrm{~A} \mathrm{~b}$ \\
\hline \multirow[t]{2}{*}{$0,45-0,55$} & $1,24 \mathrm{~A} \mathrm{a}$ & $0,92 \mathrm{C} \mathrm{a}$ & $1,11 \mathrm{~B} \mathrm{a}$ \\
\hline & \multicolumn{3}{|c|}{ Microporosidade ${ }^{* * *}\left(\mathrm{~m}^{3} \mathrm{~m}^{-3}\right)$} \\
\hline Amontoa $(-0,05-0,15)$ & $0,37 \mathrm{~A} \mathrm{c}$ & $0,35 \mathrm{~A} \mathrm{~b}$ & $0,37 \mathrm{~A} \mathrm{~b}$ \\
\hline $0,05-0,15$ & $0,39 \mathrm{~B} \mathrm{c}$ & $0,40 \mathrm{~B} \mathrm{a}$ & $0,45 \mathrm{~A}$ a \\
\hline $0,25-0,35$ & $0,44 \mathrm{Ab}$ & $0,43 \mathrm{~A}$ a & $0,43 \mathrm{~A}$ a \\
\hline \multirow{2}{*}{$0,45-0,55$} & $0,51 \mathrm{~A} \mathrm{a}$ & $0,40 \mathrm{C} \mathrm{a}$ & $0,46 \mathrm{~B}$ a \\
\hline & \multicolumn{3}{|c|}{ Macroporosidade $* * *\left(\mathrm{~m}^{3} \mathrm{~m}^{-3}\right)$} \\
\hline Amontoa $(-0,05-0,15)$ & $0,27 \mathrm{~A} \mathrm{a}$ & $0,30 \mathrm{~A} \mathrm{a}$ & $0,20 \mathrm{~B}$ a \\
\hline $0,05-0,15$ & $0,23 \mathrm{~A} \mathrm{~b}$ & $0,25 \mathrm{~A} \mathrm{~b}$ & $0,10 \mathrm{~B} \mathrm{~b}$ \\
\hline $0,25-0,35$ & $0,22 \mathrm{~A} \mathrm{~b}$ & $0,24 \mathrm{~A} \mathrm{~b}$ & $0,16 \mathrm{~B}$ a \\
\hline \multirow[t]{2}{*}{$0,45-0,55$} & $0,06 \mathrm{C} \mathrm{c}$ & $0,25 \mathrm{~A} \mathrm{~b}$ & $0,11 \mathrm{~B} \mathrm{~b}$ \\
\hline & \multicolumn{3}{|c|}{ Porosidade total $*\left(\mathrm{~m}^{3} \mathrm{~m}^{-3}\right)$} \\
\hline Amontoa $(-0,05-0,15)$ & $0,64 \mathrm{~A}$ a & $0,66 \mathrm{~A}$ a & $0,58 \mathrm{Ba}$ \\
\hline $0,05-0,15$ & $0,64 \mathrm{~A} \mathrm{a}$ & $0,65 \mathrm{~A} \mathrm{a}$ & $0,55 \mathrm{Ba}$ \\
\hline $0,25-0,35$ & $0,66 \mathrm{~A} \mathrm{a}$ & $0,67 \mathrm{~A} \mathrm{a}$ & $0,60 \mathrm{Ba}$ \\
\hline $0,45-0,55$ & $0,57 \mathrm{~B} \mathrm{~b}$ & $0,66 \mathrm{~A} \mathrm{a}$ & $0,58 \mathrm{Ba}$ \\
\hline
\end{tabular}

Médias seguidas de mesma letra minúscula na coluna e médias seguidas de mesma letra maiúscula na linha não diferem entre si pelo teste de ScottKnott $(* * * \mathrm{P}<0,001, * \mathrm{P}<0,05)$. 
O menor desenvolvimento radicular foi observado no Cambissolo na profundidade de 0,400,60 m justificado pela pior condição estrutural observada na análise visual e confirmada pela mais baixa macroporosidade observada (Tabela 3), valor inclusive bem inferior a $0,10 \mathrm{~m}^{3} \mathrm{~m}^{-3}$, considerado como referência por muitos autores (ARAÚJO JÚNIOR et al., 2011; CARDUCCI et al., 2011), e provavelmente válido para o cafeeiro quando se sabe que esta cultura é extremamente exigente em espaço de aeração do solo pelo fato de suas raízes serem desprovidas de pêlos radiculares(CARDUCCI et al., 2014).

Segundo Pereira et al. (2010), nos Cambissolos originados de rochas pelíticas é comum a presença de adensamento, o que afeta atributos morfológicos, a exemplo da consistência dura quando o solo esta seco, e físicos, a exemplo da baixa infiltração à água e baixa condutividade hidráulica (ALMEIDA; RESENDE, 1985). Nos trabalhos conduzidos por Ralisch et al. (2010), em Cambissolos sob consórcio (milho + feijão) foram observadas estruturas contínuas, coesas e de baixa porosidade, responsáveis pela baixa penetração de raízes das plantas.

Quanto aos resultados favoráveis de atributos físicos encontrados no Cambissolo até a profundidade de $0,40 \mathrm{~m}$, similares inclusive aos do Latossolo, (Tabela 3), estes se justificam pelo revolvimento efetivo, proporcionado pelo equipamento, até esta profundidade, aliviando a estrutura pelo rompimento dos agregados maiores. Somando-se a isto, há o efeito agregante posterior, proporcionado pela constante manutenção de resíduos vegetais de braquiária em diferentes níveis de decomposição, associado ao efeito do cálcio proveniente do calcário e do gesso (SILVA et al., 2016a). É importante salientar que estas informações corroboram o que já foi discutido por Silva et al. (2013), após estudo com o mesmo sistema de manejo, e Muller et al. (2012), que confirmaram efeitos positivos de doses de gesso $\left(0 ; 4 ; 8\right.$ e $12 \mathrm{Mg} \mathrm{ha}^{-}$ 1) em parâmetros relacionados com a estrutura de um Latossolo Vermelho sob plantio direto após 50 meses da aplicação, sendo observado diminuição da densidade do solo e aumentos de volume total de poros e da macroporosidade na profundidade de 0,075-0,15 m.

Ressalta-se que solos gibbsíticos, como o Latossolo do presente estudo ( $\mathrm{ki}=0,59, \mathrm{kr}=0,46)$, exibem valores de densidade do solo próximos a 1,0

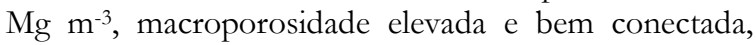
com valores superiores a $0,18 \mathrm{~cm}^{3} \mathrm{~cm}^{-3}$, como resultado da sua estrutura granular muito pequena e muito bem expressa (FERREIRA et al., 1999; ARAÚJO JÚNIOR et al., 2011; CARDUCCI et al., 2014).

\section{Análise visual da estrutura do solo}

Os baixos valores de escore visual (Ev) encontrados nas profundidades de amontoa e 0,05-0,15 $\mathrm{m}$ em todos os solos (Tabela 4) indicam boa qualidade estrutural, configurada pela presença de agregados grandes e estáveis (com muitos poros), o que favoreceu o adequado enraizamento do cafeeiro (visualmente identificado no campo), independentemente do solo avaliado.

Tabela 4 - Descrição de indicadores visuais da qualidade do solo e valores médios do Escore visual (Ev) para o CAMBISSOLO HẢPLICO, LATOSSOLO VERMELHO E NITOSSOLO HÁPLICO.

\begin{tabular}{|c|c|c|c|c|}
\hline Profundidade & Consistência do solo & Restrição às raízes & Porosidade visível & Ev \\
\hline$\ldots . .(\mathrm{m}) \ldots$. & \multicolumn{4}{|c|}{ 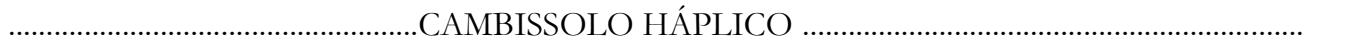 } \\
\hline amontoa & Friável & Nenhuma & Ótima/elevada & 1 \\
\hline $0,05-0,15$ & Friável & Nenhuma & Boa/moderada & 2 \\
\hline $0,25-0,35$ & Firme/Friável & Fraca & Moderadamente pobre/baixa & 3 \\
\hline \multirow[t]{2}{*}{$0,45-0,55$} & Firme/Extremamente firme & Forte & Pobre/ausente & 4 \\
\hline & \multicolumn{4}{|c|}{ VERMEHO } \\
\hline amontoa & Friável & Nenhuma & Ótima/elevada & 1 \\
\hline $0,05-0,15$ & Friável & Nenhuma & Ótima/elevada & 1 \\
\hline $0,25-0,35$ & Friável & Nenhuma & Ótima/elevada & 1 \\
\hline \multirow[t]{2}{*}{$0,45-0,55$} & Friável & Nenhuma & Boa/moderada & 2 \\
\hline & \multicolumn{4}{|c|}{ 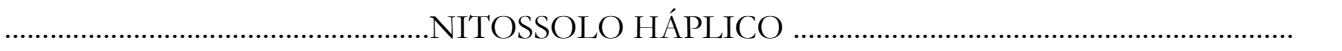 } \\
\hline amontoa & Friável & Nenhuma & Ótima/elevada & 1 \\
\hline $0,05-0,15$ & Friável & Nenhuma & Boa/moderada & 2 \\
\hline $0,25-0,35$ & Firme/Friável & Fraca & Boa/moderada & 2 \\
\hline $0,45-0,55$ & Firme/Friável & Fraca & Moderadamente pobre/baixa & 3 \\
\hline
\end{tabular}




\section{REVISTA SCIENTIA AGRARIA \\ Versão On-line ISSN 1983-2443 \\ Versão Impressa ISSN 1519-1125 \\ SA vol. $18 \mathrm{n}^{\circ} .3$ Curitiba Jul/Set. 2017 p. 61-73}

Salienta-se que a amontoa incrementou os teores de matéria orgânica na superfície do solo e isto favoreceu a agregação de partículas minerais, conforme constatado por Silva et al. (2013), o que tende a propiciar melhorias na estrutura até os $0,25 \mathrm{~m}$, particularmente no Cambissolo, que em condições naturais, é adensada (PEREIRA et al., 2010).

De maneira geral, após os cinco anos da implantação do sistema conservacionista, o Latossolo apresentou excelentes condições estruturais ao longo do perfil avaliado, o que se deve a influência da mineralogia gibbsítica $(\mathrm{ki}=0,59$, portanto $<0,75)$, que esse solo apresenta e favorece uma organização estrutural homogênea, expressa por macroestrutura do tipo granular e forte agregação dos microagregados de argila (FERREIRA et al., 1999).

A detecção de mudanças na qualidade estrutural do solo ao longo do perfil, variação de Ev (Tabela 4) é importante, por sinalizar possíveis adensamentos naturais (BALL et al., 2007), pois uma situação de $\mathrm{Ev}$ entre os valores de 4 e 5 indicam as piores condições para o desenvolvimento do sistema radicular (GUIMARÃES et al., 2011). Sendo assim, altos valores de $\mathrm{Ev}$ em subsuperfície podem indicar possibilidade de menor desenvolvimento de sistema radicular, devido à baixa presença de poros grandes. Por outro lado, é na superfície do solo que ocorre o maior ressecamento o que leva as plantas ao estresse hídrico nos períodos de ausência de água. Ball et al. (2007) estabeleceram que para as profundidades com Ev > 3 são necessárias melhorias nas práticas de cultivo para recuperação da qualidade física do solo e obtenção de maiores produtividades.

Nas profundidades de 0,25-0,35 m e 0,45-0,55 $\mathrm{m}$, o Cambissolo apresentou, pelo teste nãoparamétrico de Kruskal-Wallis $(\mathrm{P}<0,05)$, Evs médios significativamente superiores aos Evs do Latossolo. Destaca-se que na profundidade de 0,45-0,55 $\mathrm{m}$ o Cambissolo atingiu o escore 4, considerado restritivo ao adequado desenvolvimento das raízes das plantas (BALL et al., 2007). Neste caso, em primeira hipótese, sugere-se que o revolvimento somente foi efetivo até a profundidade de $0,40 \mathrm{~m}$, ou que provavelmente, ocorreu uma reacomodação das partículas do solo com o passar dos anos. Destaca-se que esta profundidade no Cambissolo se refere ao horizonte $\mathrm{Bi}$ onde $\mathrm{o}$ percentual de silte é superior a $40 \%$ (Tabela 1), e esta fração é notadamente condicionadora de adensamento, contribuindo para a consolidação do solo.

Em segunda hipótese, nas camadas mais profundas $(0,40-0,60 \mathrm{~m})$, em que o efeito da matéria orgânica é pouco expressivo (Tabela 2), a mineralogia poderá exercer o papel dominante nos processos de reorganização estrutural deste solo, logo após o seu revolvimento. Dessa forma, como nesse Cambissolo, a caulinita é o mineral dominante (Horizonte $\mathrm{Bi}(0,1-0,31$ $\mathrm{m})$ os índices de intemperismo: $\mathrm{ki}=1,45, \mathrm{kr}=1,14$, Caulinita $=50,08 \%$, Gibbsita $=13,29 \%$ ) estaria condicionando a organização estrutural típica desta classe de solo: formação de estrutura em blocos, com ajuste face a face. Em consequência, o solo se apresenta com maior densidade, maior proporção de poros pequenos e menor permeabilidade (Tabela 3) (FERREIRA et al., 1999).

\section{Relações entre análise visual da estrutura do solo e} propriedades físicas e químicas do solo

$\mathrm{O}$ método visual correlacionou-se à proporção de poros do solo de diferentes diâmetros (Tabela 5).

Tabela 5 - Coeficientes de correlação linear de Pearson para o Escore visual (Ev) e classes de diâmetro de poros $(\mu \mathrm{m})$.

\begin{tabular}{cccccc}
\hline$\varnothing>145$ & $\varnothing 145-73$ & $\varnothing 73-49$ & $\varnothing$ 49-29 & $\varnothing 29-2,9$ & $\varnothing<0,6$ \\
$-0,61 * * *$ & $-0,49 * *$ & $-0,44 * *$ & $-0,42 * *$ & $-0,39 *$ & $0,81 * * *$ \\
\hline$=$ classe de diâmetro de poros.* $\mathrm{p}<0,05,{ }^{* *} \mathrm{p}<0,01,{ }^{* * *} \mathrm{p}<0,001$. &
\end{tabular}

$\mathrm{O}$ Ev apresentou correlação positiva forte ( $\mathrm{r}$ $=0,81, \mathrm{P}<0,001)$ com a classe de poros de menor diâmetro $(<0,6 \mu \mathrm{m})$ e negativa moderada com a classe de poros de maior diâmetro $(\mathrm{r}=-0,61, \mathrm{P}<0,001)$. A porosidade visível é um dos critérios da carta padrão de AVES para definição do escore visual (GUIMARÃES et al., 2011) e estas correlações indicam que o aumento dos escores visuais esta relacionado a perda da porosidade estrutural (interagregados) e incremento da porosidade textural (intraagregados) (Tabela 5).

A forte correlação entre a classe de poros < 0,6 $\mathrm{m}$ e o escore visual atesta que a AVES pode qualificar com elevada precisão a porosidade textural. Em outras palavras, a avaliação da porosidade visível interna dos agregados naturais ou de tamanho reduzido a $1,5 \mathrm{~cm}$ pode ser utilizada com representatividade para expressar o escore visual e, consequentemente, à qualidade estrutural do solo.

Por meio dos testes de correlação de Pearson, as variáveis relacionadas com o Ev (Tabela 5) foram utilizadas na análise de componentes principais (ACP).

Verificou-se pela ACP que a distribuição das variáveis selecionadas apresentaram variância acumulada de 76,8 \% para os eixos F1 e F2, sendo que o eixo F1 foi capaz de explicar 55,2\% e o F2, $21 \%$ dessa variância (Figura 2). 
REVISTA SCIENTIA AGRARIA

Versão On-line ISSN 1983-2443

Versão Impressa ISSN 1519-1125

SA vol. $18 \mathrm{n}^{\circ} .3$ Curitiba Jul/Set. 2017 p. 61-73

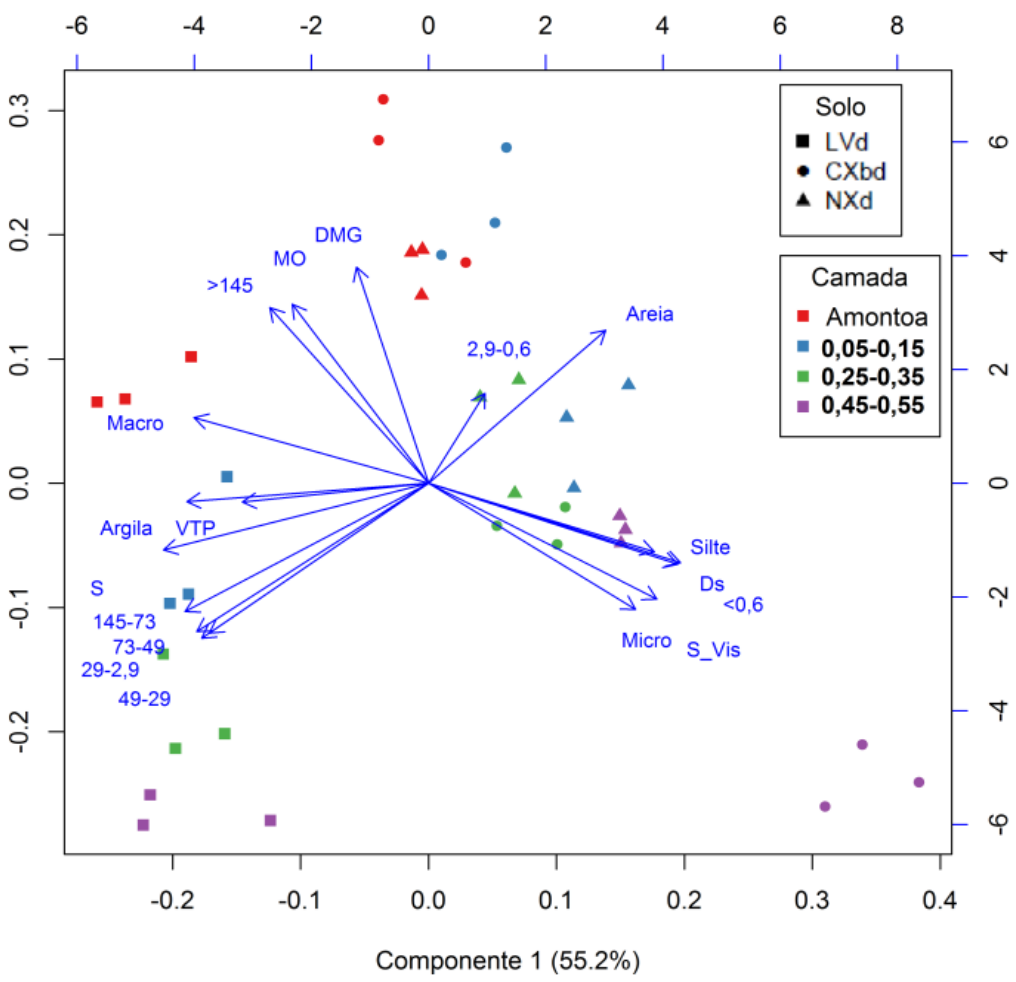

Figura 2 - Análise de componentes principais. S_Vis= Escore Visual, Ds = densidade do solo, VTP = volume total de poros, Micro $=$ microporosidade, Macro $=$ Macroporosidade, $\mathbf{D M G}=$ diâmetro médio geométrico dos agregados, classes de diâmetro de poros $(>145 ; 145-73 ; 73-49 ; 49-29 ; 29-2,9 ; 2,9-0,6 ;<0,6 \mu \mathrm{m})$, MOS = matéria orgânica do solo.

Analisando a Figura 2, depreende-se que há maior contribuição dos atributos densidade do solo, microporosidade, silte e poros da classe de diâmetro < 0,6 $\mu \mathrm{m}$ para o aumento do Ev, enquanto diâmetro médio geométrico, matéria orgânica do solo e poros da classe $>145 \mu \mathrm{m}$ explicam melhor os baixos valores de Ev. Também se observa que a amontoa está associada aos menores valores de Ev.

A profundidade de 0,45-0,55 m do Nitossolo e Cambissolo apresentou maiores restrições físicas, o que está refletido nos maiores escores visual (Tabela 4 e Figura 2). Esses resultados corroboram outros estudos relatando desenvolvimento de cafeeiros em solos rasos (SERAFIM et al., 2013), e naqueles com limitações físicas relacionadas ao adensamento pelo acúmulo de argila. No entanto para o Latossolo, as condições físicas favoráveis (Tabelas 3 e 4 ) proporcionaram presença marcante de raízes finas de absorção nessa profundidade (CARDUCCI et al., 2014, 2015).

Apesar dos importantes relatos de alguns autores (MATIELLO et al., 2002; SILVA et al., 2016b) de que a maior parte do sistema radicular ativo do cafeeiro encontra-se nos primeiros $0,30 \mathrm{~m}$, sugere-se que os estudos com raízes e sua relação com a estrutura do solo precisam ser mais criteriosos e detalhados.
Com uso de técnica de avaliação a campo e análise de imagens 2D, Carducci et al. (2014) confirmaram maior presença de raízes finas a $0,30 \mathrm{~m}$ para um Latossolo Cambissólico submetido ao mesmo sistema de manejo aqui relatado, mas o mesmo não ocorreu para $\mathrm{o}$ Latossolo típico (gibbsítico) em que as raízes do cafeeiro se apresentaram mais dispersas ao longo do perfil, demonstrando assim que estudos de diagnóstico da qualidade da estrutura do solo, desenvolvidos em campo ou laboratório, precisam levar em consideração outros aspectos relacionados ao desenvolvimento de plantas.

Neste trabalho, foram encontradas relações envolvendo $\mathrm{Ev}$ versus densidade do solo $(\mathrm{r}=0,78, \mathrm{P}<$ $0,001)$ e Ev versus matéria orgânica do solo $(\mathrm{r}=-0,82, \mathrm{P}$ $<0,001)$, resultados que corroboram aqueles encontrados por Moncada et al. (2014), que ao estudarem solos venezuelanos, também de clima tropical, encontraram que os escores obtidos pelos métodos visuais apresentaram relação com atributos físicos indicadores de qualidade do solo medidas em laboratório, como densidade do solo e carbono orgânico do solo, conforme já havia sido relatado para solos de clima temperado (GUIMARÃES et al., 2011, 2013). 
A correlação negativa entre o Ev e o teor de matéria orgânica do solo $(\mathrm{r}=-0,82, \mathrm{P}<0,001)$ evidencia que sistemas de manejo que propiciam elevado aporte de matéria orgânica, como o aqui avaliado, são desejáveis por condicionarem aumento da estabilidade dos agregados e, com isto, melhorias na estruturação do solo, provendo espaços porosos e possibilitando melhor desenvolvimento do sistema radicular das plantas, assim como aumento da atividade da fauna do solo e do fluxo de ar e água (SILVA et al., 2013).

Neste estudo, verificou-se correlação positiva entre densidade do solo versus $\mathrm{Ev}(\mathrm{r}=0,78, \mathrm{P}<0,001)$. Segundo Guimarães et al. (2011), altos valores de Ev estão associados à degradação do solo, tendo como principal agente a compactação, ou no caso do Cambissolo e Nitossolo o adensamento, que ao limitar o crescimento e desenvolvimento das raízes, pode causar redução na absorção de água e de nutrientes pelas plantas (SERAFIM et al., 2013). Para Latossolos argilosos submetidos à cafeicultura já foi sugerido que valores de densidade do solo superiores a $1,2 \mathrm{Mg} \mathrm{m}^{-3}$ poderiam limitar o crescimento radicular da cultura (ARAÚJO JÚNIOR et al., 2011), enquanto que na analise visual, Ball et al. (2007) sugeriram valores de Ev superiores a 3 como limitantes para a maioria das culturas.

Cabe destacar que, diferente deste estudo, já foram observados aumento dos escores visuais com a aplicação de gesso, mas redução da densidade do solo e incremento na porosidade total, revelando resultados contrastantes entre métodos de campo e laboratório (MULLER et al., 2012).

Considerando que no método de análise visual o principal parâmetro qualificador da qualidade estrutural se baseia numa avaliação de aspecto de agregados do solo (tamanho, resistência à ruptura, presença de raízes e porosidade visível), é possível que os resultados observados por Muller et al.(2012) sejam decorrentes das limitações que a carta padrão, proposta para o método, pode impor a avaliação de sistemas de manejo com doses de gesso acima da convencional. Pois, em Latossolo, por exemplo, os agregados do solo sob sistemas de manejo com gesso podem ser naturalmente maiores e mais resistentes à ruptura que aqueles dos seus ambientes de vegetação nativa, ou mesmo provenientes de outros sistemas de manejo. Isto porque o gesso incrementa a área dos agregados (SILVA et al., 2016a) e quando associado a elevados aportes de matéria orgânica aumenta a estabilidade dos agregados (SILVA et al., 2013).

Portanto, em solos com gesso, análises visuais, baseada na carta padrão atual, podem até indicar altos escores visuais, o que não significa que o solo esteja com sua qualidade física degradada. O aumento dos escores visuais pode ser apenas em função da escala de tamanho do agregado, que é maior, e do grau de estrutura (resistência), que é mais forte nestes ambientes.

Sabe-se também que os blocos extraídos do perfil de solo são desagregados manualmente e sua aparência é avaliada como critério de pontuação para o escore visual (BALL et al., 2007), o que significa que a porosidade estrutural (entre macroagregados), principalmente, pode ser comprometida nesse processo. Pois, conforme já comentado, a desagregação do bloco pode ser influenciada pela umidade da amostra.

Assumindo estes aspectos, as análises laboratoriais, para algumas condições em específico, são de extrema importância para complementar o diagnóstico de campo. No mais, diante das particularidades de determinados sistemas de manejo, cartas específicas podem ser necessárias, o que necessita ser mais amplamente pesquisado.

Quanto à relação entre qualidade estrutural e produtividade das plantas (Tabela 6), em levantamento preliminar, ainda não foi possível concluir quanto à viabilidade econômica do sistema de manejo. Isto porque inúmeros fatores não foram considerados, como os custos para a implantação e manutenção do sistema em cada classe de solo.

Entretanto, devido às melhorias trazidas pelo manejo aqui salientado, nas três classes de solos a média de produtividade foi bem superior à média de produção para o estado de Minas Gerais (25 sacas ha-1 - CONAB, 2013).

Tabela 6 - Produtividade das lavouras cafeeiras (em sacas/ha) em diferentes anos agrícolas e diferentes classes de solos sob manejo conservacionista.

\begin{tabular}{|c|c|c|c|c|c|}
\hline \multirow{2}{*}{ Ordem de solo } & \multicolumn{5}{|l|}{ Safra } \\
\hline & $09 / 10$ & $10 / 11$ & $11 / 12$ & $12 / 13^{*}$ & $13 / 14$ \\
\hline CAMBISSOLO HÁPLICO & 53 & 10 & 64 & - & 85 \\
\hline NITOSSOLO HÁPLICO & 69 & 10 & 69 & - & 89 \\
\hline LATOSSOLO VERMELHO & 53 & 10 & 74 & - & 75 \\
\hline
\end{tabular}

*Safra Zero (2012/2013).

Em estudos futuros de diagnóstico da qualidade da estrutura do solo, desenvolvidos em campo ou laboratório, é necessário levar em consideração aspectos gerais relacionados à parte aérea das plantas (vigor, carga pendente, tamanho dos grãos, uniformidade de maturação e rendimento), além de análises do sistema radicular (arquitetura, morfologia e área de exploração das 
raízes), no sentido de validar a relação entre qualidade estrutural com a produção vegetal.

\section{CONCLUSÕES}

A descrição visual foi eficiente na caracterização da estrutura dos solos, com resultados que corroboraram com os dados laboratoriais de densidade do solo, porosidade e agregação.

O escore visual correlacionou-se com as classes de diâmetro médio de poros do solo, principalmente poros grandes $(>145 \mu \mathrm{m})$ correlacionados aos menores escores visuais, e poros muito pequenos $(<0,6 \mu \mathrm{m})$ aos maiores escores visuais.

Os atributos densidade do solo, microporosidade, teor de silte e poros da classe de diâmetro $<0,6 \mu \mathrm{m}$ são os que mais contribuem para o aumento do escore visual, enquanto que diâmetro médio geométrico, matéria orgânica do solo e poros $>145 \mu \mathrm{m}$ justificaram os baixos valores de escore visual.

\section{AGRADECIMENTOS}

Ao Consórcio Embrapa Café, pelo empréstimo de veículos; ao CNPq e FAPEMIG, pela concessão da bolsa de estudos; à UFLA, pelo apoio institucional; à Empresa AP pelo apoio técnico e logístico.

\section{REFERÊNCIAS}

ALMEIDA, J. R.; RESENDE, M. Considerações sobre o manejo de solos raso desenvolvidos de rochas pelíticas no Estado de Minas Gerais. Informe Agropecuário, v. 11, n. 128, p. 19-26, 1985.

ARAÚJO JÚNIOR, C. F.; DIAS JUNIOR, M. S.; GUIMARÃES, P. T. C.; ALCÂNTARA, E. N. Capacidade de suporte de carga e umidade crítica de um Latossolo induzida por diferentes manejos. Revista Brasileira de Ciência do Solo, v.35, n.1, p.115131, 2011.

BALL, B.C.; WATSON, C.A.; BADDELEY, J.A. Soil physical fertility, soil structure and rooting conditions after ploughing organically managed grass/clover swards. Soil Use and Management, v.23, n.1, p. 20-27, 2007.

BALL, B.C.; DOUGLAS, J.T. A simple procedure for assessing soil structural, rooting and surface conditions. Soil Use and Management, v.19, n.1, p.5056,2003
BOUMA, J. Guide to the study of water movement in soil pedons above the watertable. Madison, University of Wisconsin, 1973. $194 \mathrm{p}$.

CARDUCCI, C. E., ZINN, Y. L., ROSSONI, D. F., HECK, R. J., OLIVEIRA, G. C. Visual analysis and $\mathrm{X}$-ray computed tomography for assessing the spatial variability of soil structure in a cultivated Oxisol. Soil and Tillage Research, v. 1, p. 12-24, 2016.

CARDUCCI, C. E.; OLIVEIRA, G. C.; CURI, N.; HECK, R. J.; ROSSONI, D.F.; CARVALHO, T. S.; COSTA, A. L. Gypsum effects on the spatial distribution of coffee roots and the pores system in oxidic Brazilian Latosol. Soil Tillage Research, p.145:171-180, 2015.

CARDUCCI, C. E.; OLIVEIRA, G. C.; CURI, N.; HECK, R. J.; ROSSONI, D. F. Scaling of pores in 3D images of Latosols (Oxisols) with contrasting mineralogy under conservation management system. Soil Research, v.52, n.3, p.231-243, 2014.

CARDUCCI, C. E.; OLIVEIRA, G. C.; SEVERIANO, E. C.; ZEVIANI, W. M. Modelagem da curva de retenção de água de Latossolos utilizando a equação duplo van Genuchten. Revista Brasileira de Ciência do Solo, v.35, n.1, p.77-86, 2011.

\section{COMPANHIA NACIONAL DE} ABASTECIMENTO-CONAB. Avaliação da Safra Agrícola Cafeeira 2013 - Segunda Estimativa Maio/2013. Disponível em: $<$ http:/ / conab.gov.br/conteudos.php?a=1253\&t $>$. Acesso: 26 julho 2013.

EMPRESA BRASILEIRA DE PESQUISA AGROPECUÁRIA - EMBRAPA. Manual de métodos de análise de solo. 2. ed. revista. Rio de Janeiro, 2011. 225p.

EMPRESA BRASILEIRA DE PESQUISA AGROPECUÁRIA - EMBRAPA. Manual de análises químicas de solos, plantas e fertilizantes. Brasília, Embrapa Informação Tecnológica; Brasília, DF: Embrapa Solos, 2009. 627p.

FERREIRA, D.F. SISVAR 5.0. Sisvar: a computer statistical analysis system. Ciência e Agrotecnologia, v.35, n.6, p.1039-1042, 2011.

FERREIRA, M. M.; FERNADES, B.; CURI, N. Mineralogia da fração argila e estrutura de Latossolos da região sudeste do Brasil. Revista Brasileira de Ciencia do Solo, v.23, n.3, p.507-514, 1999. 
GIAROLA, N. F. B; SILVA, A. P.; TORMENA, the visual evaluation of soil structure: The Brazilian experience in Oxisols under no-tillage. Soil and Tillage Research, v. 127, p. 60-64, 2013.

GIAROLA, N. F. B.; SILVA, A. P.; TORMENA, C. A.; BALL, B. \& ROSA, J. A. Visual soil structure quality assessment on Oxisols under no-tillage system. Scientia Agricola, v.67, n.4, p.479-482, 2010.

GIAROLA, N. F. B.; TORMENA, C. A.; SILVA, A. P.; BALL, B. Método de avaliação visual da qualidade da estrutura aplicado a Latossolo Vermelho Distroférrico sob diferentes sistemas de uso e manejo. Ciência Rural, v.39, n.8, p.2531-2534, 2009.

GUIMARÃES, R. M. L.; BALL, B. C.; TORMENA, C. A.; GIAROLA, N. F. B.; SILVA, A. P. Relating visual evaluation of soil structure to other physical properties in soils of contrasting texture and management. Soil and Tillage Research, v.127, p.92-99, 2013.

GUIMARÃES, P. T. G.; GARCIA, A. W. R.; ALVAREZ VIEGAS, V. H.; PREZOTTI, L. C.; VIANA, A. S.; MIGUEL, A. E.; MALAVOLTA, E.; CORREAA, J. B.; LOPES, A. S.; NOGUEIRA, F. D.; MONTEIRO, A. V. C. Cafeeiro. In: RIBEIRO, A. C.; GUIMARÃES, P. T. G.; ALVAREZ VIEGAS, V. H. (Ed.). Recomendações para o uso de corretivos e fertilizantes em Minas Gerais: 5 a aproximação. Viçosa, MG: Comissão de Fertilidade do Solo do Estado de Minas Gerais, 1999. p. 289-302.

GUIMARÃES, R. M. L.; BALL, B. C.; TORMENA, C. A. Improvements in the visual evaluation of soil structure. Soil Use and Management, v.27, p. 395-403, 2011.

JOHANNES, A.; WEISSKOPF, P.; SCHULIN, R.; BOIVIN, P. To what extent do physical measurements match with visual evaluation of soil structure? Soil and Tillage Research, 2016. http:// dx.doi.org/10.1016/j.still.2016.06.001.

MATIELLO, J. B.; SANTINATO, R.; GARCIA, A. W. R.; ALMEIDA, S. R. \& FERNADES, D. R. Cultura do café no Brasil: novo manual de recomendações. Rio de Janeiro, MAPA/Procafe, 2002. 387p.

MAZURAK, A. P. Effect of gaseous phase on water-stable synthetic aggregates. Soil Science, v.69, n.2, p.135-148, 1950.
C. A.; GUimarÃEs, R. M. L.; BALL, B. C. On MENEGASSE, L. N., GONÇALVES, J. M., FANTINEL, L. M. Disponibilidades hídricas na Província cárstica de Arcos-Pains-Doresópolis, Alto São Francisco, Minas Gerais, Brasil. Revista Águas Subterrâneas, v.16, p. 9-21, 2002.

MONCADA, P. M.; GABRIELS, D.; LOBO, D.; REY, J. C.; CORNELIS, W.M. Visual field assessment of soil structural quality in tropical soils. Soil and Tillage Research, v.139, p.8-18, 2014.

MUALEM, Y. A new model for predicting the hydraulic conductivity of unsaturated porous media. Water Resources Research, v. 12, p. 513-522, 1976.

MULLER, M. M. L.; TORMENA, C. A.; GENÚ, A. M.; KRAMER, L. F. M.; MICHALOVICZ, L.; CAÍRES, E. F. Qualidade estrutural de um Latossolo Vermelho sob plantio direto 50 meses após a aplicação de gesso. Revista Brasileira de Ciência do Solo, v.36, n.3, p.1005-1014, 2012.

PEREIRA, T. T. C.; KER, J. C.; SCHAEFER, C .E.G.R.; BARROS, N. F.; NEVES, J. C. L.; ALMEIDA, C. C. Gênese de Latossolos e Cambissolos desenvolvidos de rochas pelíticas do grupo Bambuí - Minas Gerais. Revista Brasileira de Ciência do Solo, v.34, n.4, p.1283-1295, 2010.

PRICE, N. J. P.; WHITTINGHAM, M. J.; CHAMBERS, B. J.; PEEL. S. Visual soil evaluation in relation to measured soil physical properties in a survey of grassland soil compaction in England and Wales. Soil and Tillage Research, v.127, p.65-73, 2013.

R Development Core Team. R: a language and environment for statistical computing. 2013. Avaiable at Institute for Statistics and Mathematics. Vienna, Gemany. Disponível em: <http://www.Rproject.org >. Acessado em: 15 outubro 2013.

RALISCH, R.; ALMEIDA, E.; SILVA, A. P.; NETO, O.C.P.; GUIMARÃES, M. F. Morphostructural characterization of soil conventionally tilled with mechanized and animal traction with and without cover crop. Revista Brasileira de Ciência do Solo, v.34, n.6, p.1795-1802, 2010.

SANTOS, H. G. dos; JACOMINE, P. K. T.; ANJOS, L. H. C. dos; OLIVEIRA, V. A. de; LUMBRERAS, J. F.; COELHO, M. R.; ALMEIDA, J. A. de; CUNHA, T. J. F.; OLIVEIRA, J. B. de. Sistema brasileiro de classificação de solos. 3. ed. rev. e ampl. Brasília, DF: Embrapa, 2013. 353 p. 
SERAFIM, M. E.; OLIVEIRA, G. C.; CURI, N.; LIMA, J. M.; GUIMARÃES, P. T. G.; LIMA, V. M. P. Potencialidades e limitações de uso de Latossolos e Cambissolos, sob sistema conservacionista em lavouras cafeeiras. Bioscience Journal, v.29, n.5, p.1640-1652, 2013.

SERAFIM, M. E.; OLIVEIRA, G. C.; OLIVEIRA, A. S.; LIMA, J. M.; GUIMARÃES, P. T. G.; COSTA, J. C. Sistema conservacionista e de manejo intensivo do solo no cultivo de cafeeiros na região do Alto São Francísco, MG: um estudo de caso. Bioscience Journal, v. 27, n.6, p.964-977, 2011.

SILVA, É. A.; OLIVEIRA, G. C.; CARDUCCI, C. E.; SILVA, B. M.; Serafim, M.E. Aggregates morphometry of a Inceptisol under conservationist system. Semina: Ciências Agrárias, v. 37, n. 3, p. 1165-1176, 2016a.

SILVA, E. A.; SILVA, S. H. G.; OLIVEIRA, G. C.; CARDUCCI, C. E. Root spatial distribution in coffee plants of different ages under conservation management system. African Journal of Agricultural Research, v.11, p.4970-4978, 2016 b.

SILVA, E. A.; OLIVEIRA, G. C.; CARDUCCI, C. E.; SILVA, B. M.; OLIVEIRA, L. M.; COSTA, J. C. Increasing doses of agricultural gypsum, aggregate stability and organic carbon in Cerrado Latosol under coffee crop. Revista Ciências Agrárias, v.56, n.1, p.25-32, 2013.

TAVARES FILHO, J.; RALISCH, R.; GUIMARÃES, M. F.; MEDINA, C. C.; BALBINO, L. C.; NEVES, C. S. V. J. Método do perfil cultural para avaliação do estado físico de solos em condições tropicais. Revista Brasileira de Ciência do Solo, v.23, n.2, p.393-399, 1999.

van GENUCHTEN, M. T. A. A closed-form equation for predicting the hydraulic conductivity of unsaturated soils. Soil Science Society of America Journal, v. 44, p. 892-898, 1980. 